\title{
Curvature and rank of Teichmüller space
}

\author{
Jeffrey Brock* and Benson Farb ${ }^{\dagger}$
}

September 6, 2001

\begin{abstract}
Let $S$ be a surface with genus $g$ and $n$ boundary components and let $d(S)=3 g-3+n$ denote the number of curves in any pants decomposition of $S$. We employ metric properties of the graph of pants decompositions $C_{\mathbf{P}}(S)$ prove that the Weil-Petersson metric on Teichmüller space Teich $(S)$ is Gromov-hyperbolic if and only if $d(S) \leq 2$. When $d(S) \geq 3$ the Weil-Petersson metric has higher rank in the sense of Gromov (it admits a quasi-isometric embedding of $\mathbb{R}^{k}, k \geq 2$ ); when $d(S) \leq 2$ we combine the hyperbolicity of the complex of curves and the relative hyperbolicity of $C_{\mathbf{P}}(S)$ prove Gromov-hyperbolicity.

We prove moreover that Teich $(S)$ admits no geodesically complete Gromov-hyperbolic metric of finite covolume when $d(S) \geq 3$, and that no complete Riemannian metric of pinched negative curvature exists on Moduli space $\mathcal{M}(S)$ when $d(S) \geq 2$.
\end{abstract}

\section{Introduction}

The Weil-Petersson metric on Teichmüller space Teich $(S)$ has many curious properties. It is a Riemannian metric with negative sectional curvature, but its curvatures are not bounded away from zero or negative infinity. It is geodesically convex, but it is not complete. In this paper we show that in spite of exhibiting negative curvature behavior, the Weil-Petersson metric is not coarsely negatively curved except for topologically simple surfaces $S$. Our main theorem answers a question of Bowditch [Be, Question 11.4].

Theorem 1.1 Let $S$ be a compact surface of genus $g$ with $n$ boundary components. Then the Weil-Petersson metric on Teich $(S)$ is Gromov-hyperbolic if and only if $3 g-3+n \leq 2$.

\footnotetext{
${ }^{*}$ Research supported by the NSF.

${ }^{\dagger}$ Research supported by the NSF and the Sloan Foundation.
} 
The constant $d(S)=3 g-3+n$ is fundamental in Teichmüller theory: it is the complex dimension of the Teichmüller space Teich $(S)$, or more topologically, the number of curves in any pair-of-pants decomposition of $S$. An equivalent formulation of Theorem 1.1, then, is that the Weil-Petersson metric is Gromov-hyperbolic precisely when the interior $\operatorname{int}(S)$ is a torus with at most two punctures or a sphere with at most five punctures.

Theorem 1.1 exhibits the first example of a $(\operatorname{Mod}(S)$-invariant) metric on a Teichmüller space of (real) dimension greater than 2 that is Gromovhyperbolic. In contrast, these Teichmüller spaces admit no complete Riemannian metric of pinched negative sectional curvature (Theorem 1.3 below). To summarize, the overlap of the positive and negative results in this paper give:

When $d(S)=2$, the Weil-Petersson metic on Teich $(S)$ is Gromov-hyperbolic, yet $\operatorname{Teich}(S)$ admits no (equivariant) complete, Riemannian metric with pinched negative curvature.

The fact that the Weil-Petersson metric is not Gromov-hyperbolic when $d(S) \geq 3$ relies heavily on a geometric investigation of a combinatorial model for the Weil-Petersson metric constructed in [Br] (see below).

Constraints on metrics on $\mathcal{M}_{\boldsymbol{g}, \boldsymbol{n}}$. In S. Kravetz' 1959 paper [Kr], it was claimed that the Teichmüller metric on the moduli space $\mathcal{M}_{g, n}$ of Riemann surfaces of genus $g \geq 2$ with $n$ punctures is a complete metric with negative curvature in the sense of Busemann, i.e. that $\operatorname{Teich}\left(S_{g, n}\right)$ admits such an equivarient metric. The proof had an error, and the result was shown to be false in [Mas1]; in fact Masur-Wolf showed in [MW] that the Teichmüller metric is not even Gromov-hyperbolic (see [MP1] and [MP2] for two other proofs). The following theorem shows that this phenomenon has little to do with the Teichmüller metric; in fact it holds for a broad class of metrics.

We say that a geodesically complete metric space $X$ has finite volume if for each $\epsilon>0$ there is no infinite collection of pairwise disjoint $\epsilon$-balls embedded in $X$.

Theorem 1.2 Supposed $(S) \geq 3$. Then Teich $(S)$ admits no proper, geodesically complete, Gromov-hyperbolic $\operatorname{Mod}(S)$-equivariant path metric with finite covolume.

Theorem 1.2 applies in particular to the Teichmüller metric, which satisfies the finite volume condition, giving the main result of $[\mathrm{MW}]$ when $d(S) \geq$ 3. Theorem 1.2 also applies to McMullen's Kähler hyperbolic metric $h$ on $\operatorname{Teich}(S)$ constructed in $[\mathrm{Mc}]$ as it is complete and Riemannian, and has 
finite volume quotient on $\mathcal{M}_{g, n}$ (it is also quasi-isometric to the Teichmüller metric).

We note that our argument for Theorem 1.2 breaks down in the case of the Weil-Petersson metric, which is not complete as a metric space (and in particular not geodesically complete). Indeed, with the isometric action of $\operatorname{Mod}(S)$ on the Weil-Petersson metric, Dehn twists are infinite order elliptic elements, as they act with bounded orbits. The first part of our proof of Theorem 1.2 is essentially an argument of McCarthy-Papadopoulos [MP1], where the theorem is proven under the additional hypothesis that every pseudo-Anosov element acts as a hyperbolic isometry. We reproduce the argument here for completeness and since it is brief; we also extend the proof to the punctured case.

In the absence of the finite-volume hypothesis, it is possible to say something about complete Riemannian metrics of pinched negative curvature.

Theorem 1.3 If $3 g-3+n \geq 2$ then $\mathcal{M}_{g, n}$ admits no complete Riemannian metric of pinched negative sectional curvature.

Note that Theorem 1.2 implies Theorem 1.3 in the finite volume case for $d(S) \geq 3$ but does not cover the case when $d(S)=2$, i.e. when $\operatorname{int}(S)$ is a torus with two punctures or a sphere with five punctures.

The rank of the Weil-Petersson metric. To prove that the WeilPetersson metric is not Gromov-hyperbolic when $d(S) \geq 3$ (the "only if" part of Theorem 1.1), we show it has higher rank in these cases.

The rank of a metric space $X$ is the maximal dimension $n$ of a quasi-flat in $X$, that is a quasi-isometric embedding $\mathbb{R}^{n} \rightarrow X$. This notion of rank was introduced by Gromov ([Grom], 6. $B_{2}$ ), and agrees with the usual notion of rank of a nonpositively curved symmetric space (this follows easily from the quasi-flats theorem of $[\mathrm{EF}]$ and $[\mathrm{KL}]$ ).

There has been a recurring comparison in the literature of Teichmüller space to symmetric spaces of noncompact type, particularly in terms of the rank one/higher rank dichotomy (see, e.g., [Iv1, Iv2, FLM]). The following theorem adds to the list of higher rank behavior of Teichmüller space.

Theorem 1.4 The rank of the Weil-Petersson metric on Teich $(S)$ is at least $d(S) / 2$.

As Gromov-hyperbolic metric spaces have rank one, Theorem 1.4 implies one direction of Theorem 1.1. We conjecture that the Weil-Petersson metric on Teich $(S)$ has rank precisely the integer part of $(d(S)+1) / 2$; the conjecture 
is supported by the hierarchies machinery of [MM2], but bounding the rank from above appears delicate.

Remark. While Dehn twists about disjoint simple closed curves produce quasi-flats in the mapping class group (endowed with the word metric) see $[\mathrm{FLM}]$, Dehn twist orbits in $\operatorname{Teich}(S)$ do not generate quasi-flats in either the Weil-Petersson or Teichmüller metrics: indeed, if $\tau \in \operatorname{Mod}(S)$ is a Dehn twist, we have $d_{\mathrm{WP}}\left(X, \tau^{n} X\right)=O(1)$ and $d_{\mathrm{T}}\left(X, \tau^{n} X\right)=O(\log (n))$. Evidently, the appearance of an orbit of a subgroup generated by commuting Dehn twists in the Teichmüller metric is more akin to a horosphere in a rank-one symmetric space. (Note that in higher rank symmetric spaces horospheres are actually quasi-isometrically embedded.)

Combinatorics of curves on surfaces. The proof of Theorems 1.1 and 1.4 rely on important work of Masur and Minsky [MM1, MM2] on combinatorial complexes associated to curves on surfaces.

Let $\mathcal{S}$ denote the set of all isotopy classes of essential, non-peripheral, simple closed curves on the surface $S$. The curve complex $\mathcal{C}(S)$ is the simplicial complex whose vertices are the elements of $\mathcal{S}$ and whose $k$-simplices span collections of $k+1$ curves in $\mathcal{S}$ that can be realized pairwise disjointly on $S$. Metrizing each simplex to be the standard Euclidean simplex, one obtains a metric on $\mathcal{C}(S)$. The main theorem of [MM1] is that $\mathcal{C}(S)$ endowed with this metric is a Gromov-hyperbolic metric space.

Our proof of Theorem 1.4 uses a closely related combinatorial object. Consider the graph whose vertices are pair-of-pants decompositions of $S$, and whose edges join decompositions that differ by a single elementary move (see Figure 1). Assigning each edge length 1, we obtain a graph $C_{\mathbf{P}}(S)$ with a distance function $d_{\mathbf{P}}(.,$.$) on pairs of vertices given by taking the minimal$ length path between two pants-decompositions. This graph is the 1-skeleton of a simplicial complex introduced by Hatcher-Thurston [HT]; in particular they proved that this graph is connected. The graph $C_{\mathbf{P}}(S)$ coarsely models Weil-Petersson geometry.

Theorem 1.5 ( $[\mathrm{Br}]$ ) The graph $C_{\mathbf{P}}(S)$ is quasi-isometric to Teich $(S)$ endowed with the Weil-Petersson metric.

The proof of Theorem 1.4 can thus be reduced to finding quasi-isometrically embedded flats in the graph $C_{\mathbf{P}}(S)$. The hypothesis is explained by the need for at least two disjoint essential subsurfaces of $S$ whose Teichmüller spaces are themselves non-trivial, in which case flats arise from pants decompositions related by elementary moves that occur in disjoint subsurfaces. 
Figure 1. Elementary moves on pants decompositions.

Conversely, when $d(S) \leq 2$, one cannot perform independent elementary moves on pairs of pants because there are not enough disjoint subsurfaces on which to perform them. The lack of available subsurfaces leads one to consider the possibility that $C_{\mathbf{P}}(S)$ is Gromov-hyperbolicity in these cases. Gromov-hyperbolicity is known for $d(S)=1$ by [MM1]; we establish Gromov-hyperbolicity of $C_{\mathbf{P}}(S)$ for for $d(S)=2$ using three ingredients: hyperbolicity of the complex of curves $\mathcal{C}(S)$ (proved in [MM1]), the theory of relative hyperbolicity developed in $[\mathrm{Fa}]$, and the hierarchical structure of $\mathcal{C}(S)$ given in [MM2].

Plan of the paper. Section 2 gives preliminaries on Teichmüller theory and Weil-Petersson geometry, Gromov-hyperbolic metric spaces, and the combinatorics of curves on surfaces. Section 3 contains the proof of nonexistence of geodesically complete Gromov-hyperbolic metrics on Teich $(S)$ with finite covolume when $d(S) \geq 3$ (Theorem 1.2), and gives constraints on complete Riemannian metrics of pinched negative curvature on $\mathcal{M}_{g, n}$ (Theorem 1.3).

In section 4 we show that the Weil-Petersson metric has higher rank when $d(S) \geq 3$ (Theorem 1.4), proving one direction of Theorem 1.1. Finally, in Section 5 we prove the other direction of Theorem 1.1 by showing the WeilPetersson metric is Gromov-hyperbolic when $d(S) \leq 2$.

We conclude the paper with a list of questions for further investigation. 
Ackhowledgements. The authors would like to thank Howard Masur and Yair Minsky for many informative discussions on the topic of this paper.

\section{Preliminaries}

\subsection{Teichmüller space}

Let $S$ be a topological surface, possibly with boundary. When $S$ has boundary $\partial S$ denote by $\operatorname{int}(S)$ its interior $S-\partial S$. The Teichmüller space Teich $(S)$ parameterizes finite area hyperbolic surfaces $X$ equipped with markings, or homeomorphisms $(f: \operatorname{int}(S) \rightarrow X)$ up to isometries that preserve the marking: i.e.

$$
(f: \operatorname{int}(S) \rightarrow X) \sim(g: \operatorname{int}(S) \rightarrow Y)
$$

if there is an isometry $\phi: X \rightarrow Y$ so that $g \simeq \phi \circ f$.

The space Teich $(S)$ is topologized by the distance

$$
d((f, X),(g, Y))=\inf _{\varphi} \log L(\varphi)
$$

where the infimum is taken over all bi-Lipschitz diffeomorphisms $\varphi$ isotopic to $g \circ f^{-1}$ and $L(\varphi)$ is the minimal bi-Lipschitz constant for $\varphi$. It is homeomorphic to a cell of dimension $6 g-6+2 n$ where $n$ is the number of boundary components of $S$.

The Teichmüller space carries a natural complex structure; its complex cotangent space $T_{X}^{*} \operatorname{Teich}(S)$ at $X \in \operatorname{Teich}(S)$ is identified with the space of holomorphic quadratic differentials $Q(X)$ on $X$. The Weil-Petersson metric on Teich $(S)$ is obtained by duality from the $L^{2}$-inner product on $Q(X)$

$$
\langle\varphi, \psi\rangle_{\mathrm{WP}}=\left(\int_{X} \frac{\varphi \bar{\psi}}{\rho^{2}}|d z|^{2}\right)^{1 / 2}
$$

where $\rho(z)|d z|$ is the hyperbolic line element on $X$.

\subsection{The pants complex}

Let $\mathcal{S}$ denote the isotopy classes of essential simple closed curves on $S$. A pants decomposition $P$ of $S$ is a maximal collection of distinct elements of $\mathcal{S}$ so that no two isotopy classes in $P$ have representatives that intersect. The pants complex $C_{\mathbf{P}}(S)$ is the graph with one vertex for each pants decomposition and an edge joining each pair of vertices whose pants decompositions differ by an elementary move (see figure 1). The distance function

$$
d_{\mathbf{P}}: C_{\mathbf{P}}^{0}(S) \times C_{\mathbf{P}}^{0}(S) \rightarrow \mathbb{Z}_{\geq 0}
$$


on the vertex set $C_{\mathbf{P}}^{0}(S)$ of $C_{\mathbf{P}}(S)$ counts the minimal number of elementary moves between maximal partitions.

Geodesic representatives. We briefly describe the quasi-isometry of Theorem 1.5.

Given $X \in \operatorname{Teich}(S)$, the elements of $P$ can be represented as pairwise disjoint closed geodesics on $X$. The sub-level sets for the lengths of the elements of $P$ play a special role in Weil-Petersson geometry. Let

$$
V_{L}(P)=\left\{X \in \operatorname{Teich}(S) \mid \ell_{X}(\alpha)<L \text { for each } \alpha \in P\right\} .
$$

Applying theorems of Wolpert [Wol] and Masur [Mas2], we have the following (see $[\mathrm{Br}])$ :

Proposition 2.1 Given $L$ there is a $D_{L}$ so that for each $P \in C_{\mathbf{P}}^{0}(S)$, the sub-level set has Weil-Petersson diameter

$$
\operatorname{diam}_{W P}\left(V_{L}(P)\right)<D_{L}
$$

By a theorem of Bers, there is an $L>0$ so that the union

$$
\bigcup_{P \in C_{\mathbf{P}}^{0}(S)} V_{L}(P)
$$

covers Teich $(S)$. Let $V(P)=V_{2 L}(P)$. Let

$$
Q: C_{\mathbf{P}}^{0}(S) \rightarrow \operatorname{Teich}(S)
$$

be any map for which $Q(P) \in V(P)$. Then Theorem 1.1 of $[\mathrm{Br}]$ shows $Q$ is a quasi-isometry. In other words there are constants $K_{1}>1$ and $K_{2}>0$ depending only on $S$ so that given $X \in V\left(P_{X}\right)$ and $Y \in V\left(P_{Y}\right)$, we have

$$
\frac{1}{K_{1}} d_{\mathbf{P}}\left(P_{X}, P_{Y}\right)-K_{2} \leq d_{\mathrm{WP}}(X, Y) \leq d_{\mathbf{P}}\left(P_{X}, P_{Y}\right)+K_{2} .
$$

\subsection{The curve complex}

Let $\mathcal{C}(S)$ be the complex associated to the simple closed curves $\mathcal{S}$ on $S$ as follows:

- The zero-skeleton $\mathcal{C}^{0}(S)$ is identified with the elements of $\mathcal{S}$.

- Any $k+1$ curves $\left(\alpha_{1}, \ldots, \alpha_{k+1}\right)$ in $\mathcal{S}^{k+1}$ with the property $\alpha_{i} \neq \alpha_{j}$ and $i\left(\alpha_{i}, \alpha_{j}\right)=0$, for $i \neq j$ determine a $k$ simplex in $\mathcal{C}(S)$. 
For an essential, non-annular subsurface $Y \subset S$, the curve complex $\mathcal{C}(Y)$ is a subcomplex of $\mathcal{C}(S)$. The subsurface projection

$$
\pi_{Y}: \mathcal{C}(S) \rightarrow \mathcal{P}(\mathcal{C}(Y))
$$

from the curve complex $\mathcal{C}(S)$ to the set $\mathcal{P}(\mathcal{C}(Y))$ of all subsets of $\mathcal{C}(Y)$ is defined by setting $\pi_{Y}(\alpha)=\alpha$ if $\alpha \in \mathcal{C}(Y)$ and letting

$$
\pi_{Y}(\alpha)=\cup_{\alpha^{\prime}} \partial \mathcal{N}\left(\alpha^{\prime} \cup \partial_{\alpha^{\prime}} Y\right)
$$

be the union over all $\operatorname{arcs} \alpha^{\prime}$ of essential intersection of $\alpha$ with $Y$ of a regular neighborhood $\partial \mathcal{N}\left(\alpha^{\prime} \cup \partial_{\alpha^{\prime}} Y\right)$ of $\alpha^{\prime} \cup \partial_{\alpha^{\prime}} Y$, where $\partial_{\alpha^{\prime}} Y$ represents the components of the boundary of $Y$ that $\alpha^{\prime}$ intersects (see [MM2, Sec. 2]).

For two subsets $A$ and $B$ of $\mathcal{C}(Y)$, the (semi)-distance $d_{Y}(A, B)$ is defined by

$$
d_{Y}(A, B)=\operatorname{diam}_{\mathcal{C}(Y)}(A \cup B) .
$$

This notion of distance allows us to measure the distance between pants decompositions $P$ and $P^{\prime}$ relative to $Y$ by letting $\pi_{Y}(P)=\cup_{\alpha \in P} \pi_{Y}(\alpha)$ and letting the projection distance $d_{Y}\left(P, P^{\prime}\right)$ between $P$ and $P^{\prime}$ relative to $Y$ be

$$
d_{Y}\left(P, P^{\prime}\right)=d_{Y}\left(\pi_{Y}(P), \pi_{Y}\left(P^{\prime}\right)\right) .
$$

We also record for reference the following Lipschitz property for the projection $\pi_{Y}$ :

Lemma 2.2 (Lem. 2.3 of [MM2]) Let $Y$ be an essential subsurface of $S$. Then if $\alpha$ and $\beta$ simple closed curves whose vertices have distance one in $\mathcal{C}(S)$ and $\pi_{Y}(\alpha) \neq \varnothing \neq \pi_{Y}(\beta)$, then we have $d_{Y}(\alpha, \beta) \leq 2$.

For a detailed discussion of the curve complex and related projection mappings, see [MM1] and [MM2]

\subsection{Hyperbolic metric spaces}

In this subsection we briefly recall some material on hyperbolic metric spaces. The standard reference for this material is $[\mathrm{GH}]$. All statements about hyperbolic metric spaces which we use can be found in $[\mathrm{GH}]$.

A metric space $X$ is proper if every closed ball in $X$ is compact. If for any $x, y \in X$, there exists a parametrized path $\gamma:[0, d(x, y)] \rightarrow X$ from $x$ to $y$ with $d(\gamma(s), \gamma(t))=|s-t|$ for all $s, t \in[0, d(x, y)]$ then $X$ is called a geodesic metric space. Here we are using the metric space notion of path length. 
$X$ is a Gromov-hyperbolic, or $\delta$-hyperbolic metric space if there exists $\delta>0$ so that every geodesic triangle $T$ in $X$ is $\delta$-thin: the $\delta$-neighborhood of any two sides of $T$ contains the third side.

A $\delta$-hyperbolic metric space has a natural compactification $X \cup \partial X$ where $\partial X$ is the collection of Hausdorff equivalence classes of geodesic rays in $X$. Every isometry of $X$ acts by homeomorphisms on $\partial X$. We denote the fixed set of the action of an isometry $g \in \operatorname{Isom}(X)$ in $\partial X$ by $\operatorname{Fix}(g)$.

Combining Theorems 16 and 17 of Section 8 of [GH] gives the following classification theorem.

Theorem 2.3 (Classification of isometries) Every isometry $g$ of a $\delta$ hyperbolic metric space is precisely one of the following types:

1. elliptic: every g-orbit is bounded.

2. hyperbolic: $\operatorname{Fix}(g)=\{x, y\}$ for some $x \neq y$ in $\partial X$, in which case any $g$-orbit in $X$ is a quasi-geodesic with limit set $\{x, y\}$.

3. parabolic: $\operatorname{Fix}(g)=\{x\}$ for some $x \in \partial X$.

\subsection{The rank of a metric space}

A metric space has a quasi-flat of dimension $n$ if there is a quasi-isometric embedding $F: \mathbb{R}^{n} \rightarrow X$. We say a metric space has higher rank if it admits a quasi-flat of dimension at least 2 . Since $\mathbb{R}^{n}$ is not Gromov-hyperbolic, the quasi-isometric embedding $F$ provides a family of triangles in $X$ that violates the $\delta$-thin condition for all $\delta>0$. Thus, a higher rank metric space is not Gromov-hyperbolic.

\section{Constraints on metrics on $\mathcal{M}_{g, n}$}

In this section we prove Theorems 1.2 and 1.3.

The idea of Theorem 1.2 is that a properly discontinuous action of $\operatorname{Mod}(S)$ on a geodesically complete, $\delta$-hyperbolic metric space has certain special properties when $S$ is sufficiently complicated. Indeed, when $d(S) \geq 3$, then entire group $\operatorname{Mod}(S)$ must act parabolically with a single parabolic fixed point at infinity. This first part is essentially an argument of McCarthyPapadopoulos [MP2]. As with isometric actions on hyperbolic space, we show that such an action cannot have finite volume quotient.

Notation: For notational purposes in the following arguments, we will use $S_{g, n}$ to refer to a surface with genus $g$ and $n$ boundary components. 
Proof: (of Theorem 1.2). Suppose to the contrary that $X=\operatorname{Teich}\left(S_{g, n}\right)$ admits a $\operatorname{Mod}\left(S_{g, n}\right)$-equivariant, geodesically complete path metric which is $\delta$-hyperbolic. We have that $\operatorname{Mod}\left(S_{g, n}\right)$ acts properly discontinuously on $X$ by isometries, and $($ see $\S 2.4)$ that $\operatorname{Mod}\left(S_{g, n}\right)$ thus acts by homeomorphisms on the Gromov boundary $\partial X$.

We claim that since $3 g-3+n \geq 3$, we may pick a generating set $\left\{g_{i}\right\}$ for $\operatorname{Mod}\left(S_{g, n}\right)$ consisting of Dehn twists about non-separating curves, with the following properties:

1. Each $g_{i}$ is conjugate in $\operatorname{Mod}\left(S_{g, n}\right)$ to each $g_{j}$.

2. The group generated by elements commuting with $g_{1}$ is not virtually cyclic, i.e. it does not contain a cyclic subgroup of finite index; similary for $g_{2}$.

3. $g_{1}$ and $g_{2}$ do not commute; in fact sufficiently high powers of $g_{1}$ and $g_{2}$ generate a free group.

4. The commuting graph for $\left\{g_{i}\right\}$, consisting of a vertex for each $g_{i}$ and an edge connecting commuting elements, is connected.

When $n=0$ one may take the "standard" Dehn-Lickorish-Humphries generators (see $[\mathrm{FF}]$ ). For $n>0$ one proceeds inductively by using the exact sequence (see, e.g. [Iv2]):

$$
1 \rightarrow \pi_{1}\left(S_{g, n}\right) \rightarrow \operatorname{Mod}\left(S_{g, n+1}\right) \rightarrow \operatorname{Mod}\left(S_{g, n}\right) \rightarrow 1
$$

where the kernel is generated by "pushing the puncture" around a given loop, one for each loop in a standard generating set for $\pi_{1}\left(S_{g, n}\right)$. Such elements of $\operatorname{Mod}\left(S_{g, n+1}\right)$ are generated by elements $\alpha_{1} \alpha_{2}^{-1}$ where each $\alpha_{i}$ is a Dehn twist about a non-separating curve; one then adds these Dehn twists to the previous list of generators, easily checking the required properties, and continues inductively.

The elements $g_{1}$ and $g_{2}$ may then be taken to be an intersecting pair of loops in the Dehn-Humphries-Lickorish generating set. In particular, the group generated by elements commuting with $g_{i}$ (for $i=1$ or $i=2$ ), contains the mapping class group $\operatorname{Mod}\left(S_{g, n-1}\right)$, which is not virtually cyclic for $3 g-3+n \geq 3$.

Now apply the classification of isometries of $\delta$-hyperbolic metric spaces (see Theorem 2.3 above) to $g_{1}$. Note that this classification uses the geodesic assumption on the metric space $X$. As $g_{1}$ has infinite order and the action of $\operatorname{Mod}\left(S_{g, n}\right)$ is properly discontinuous, it follows that $g_{1}$ is not of elliptic 
type. Suppose $g_{1}$ is of hyperbolic type. Then $\operatorname{Fix}\left(g_{1}\right)=\{x, y\}$ for some $x \neq y$ in $\partial X$.

The subgroup $H$ of $\operatorname{Mod}\left(S_{g, n}\right)$ commuting with $g_{1}$ clearly leaves $\{x, y\}$ invariant, and is not virtually cyclic. But Theorem 30 in Section 8 of [GH] states that, for a group $\Gamma$ acting properly discontinuously on a proper, geodesic, $\delta$-hyperbolic metric space $X$, the stabilizer of a pair of distinct points $\{x, y\}$ has a cyclic subgroup of finite index, a contradiction. Thus it must be that $g_{1}$ is of parabolic type, and so $\operatorname{Fix}\left(g_{1}\right)=x$ for some $x \in \partial X$.

As each $g_{i}$ is conjugate to $g_{1}$, each $g_{i}$ is also of parabolic type, say fixing the unique point $x_{i} \in \partial X$. As Dehn twists about disjoint curves commute, since $[g, h]=1$ implies $g(\operatorname{Fix}(h))=\operatorname{Fix}(h)$, and since the commuting graph of $\left\{g_{i}\right\}$ is connected, it follows that $\operatorname{Fix}(g)=x$ for each $g \in\left\{g_{i}\right\}$.

We claim that every element of $\operatorname{Mod}\left(S_{g, n}\right)$, not just the generating set, is of parabolic type with unique fixed point $x \in \partial X$. As $\operatorname{Mod}\left(S_{g, n}\right)$ has a torsion-free subgroup of finite index, if this is not true then there exists $g \in \operatorname{Mod}\left(S_{g, n}\right)$ acting on $X$ as an isometry of hyperbolic type, with $x$ as an attracting point. Pick any $z \in X$. By Theorem 8.21 of [GH], the orbit $\left\{g^{n} z: n \in \mathbb{Z}\right\}$ is a $K$-quasigeodesic in $x$ for some $K \geq 1$ and has limit point $x$ as $n \rightarrow \infty$. Pick any element $h \in \operatorname{Mod}\left(S_{g, n}\right)$ acting as a parabolic fixing $x$ (such $h$ exist by the previous paragraph). Since $\left\{g^{n} z: n \in \mathbb{Z}\right\}$ and $\left\{h g^{n} z: n \in \mathbb{Z}\right\}$ are both $K$-quasigeodesics which limit to $x$ as $n \rightarrow \infty$, it follows that there exists $N>0, C>0$ so that $d\left(g^{n}(z), h g^{n}(z)\right)<C$ for all $n>N$. But then $d\left(g^{-n} h g^{n}(z), z\right)=d\left(h g^{n}(z), g^{n}(z)\right)<C$ for all $n>N$. Since $h$ is of parabolic type and $g$ is of hyperbolic type, the set $\left\{g^{-n} h g^{n}: n \in \mathbb{Z}\right\}$ is infinite, so that the hypothesis of proper discontinuity is violated. Hence the claim is proved ${ }^{1}$.

It follows that $\operatorname{Mod}\left(S_{g, n}\right)$ permutes the set of equivalence classes of geodesic rays with $x$ as their common endpoint at infinity $x \in \partial X$. We now show that one of the hypotheses of the theorem must be violated.

Recall from $\S 8.1$ of $[\mathrm{GH}]$ that any choice of a point $x \in \partial X$ and basepoint $y \in X$ determines a Busemann function $\beta: X \rightarrow \mathbb{R}$ on $X$ defined by

$$
\left.\beta(z)=\sup _{\gamma}\left\{\limsup _{t \rightarrow \infty}\left(d_{X}(z, \gamma(t))-t\right)\right)\right\}
$$

where the sup is taken over all geodesic rays $\gamma$ based at $y$ with $\gamma(\infty)=x$. In the proof of Proposition 8.18 of [GH] (see also Remark 8.13.ii) it is shown that any parabolic isometry $g$ fixing $x \in \partial X$ must almost preserve level

\footnotetext{
${ }^{1}$ While this claim is obviously true in the negatively curved Riemannian context without the proper discontinuity hypothesis, it may not be true without it for arbitrary Gromov-hyperbolic spaces; see [GH], 8.13.
} 
sets of the Busemann function, that is there exists a constant $C$ so that $\left|\beta(w)-\beta\left(g^{n}(w)\right)\right| \leq C$ for all $n \in \mathbb{Z}$ and any $w$ lying on the ray $\gamma$.

As this holds true for all $g \in \operatorname{Mod}\left(S_{g, n}\right)$, it follows that any $\operatorname{Mod}\left(S_{g, n}\right)$ orbit in $X$ lies within a bounded distance of some level set of $\beta$. But $\beta$ is clearly proper on $\gamma$; in particular there exists a constant $\epsilon$ and points $z_{i}, i=1,2, \ldots$ on $\gamma$ with the property that for any $i, j$ with $i \neq j$, the $\epsilon$-ball centered at $z_{i}$ is disjoint from the $\operatorname{Mod}\left(S_{g, n}\right)$-orbit of the $\epsilon$-ball centered at $z_{j}$. In particular the quotient $\operatorname{Teich}\left(S_{g, n}\right) / \operatorname{Mod}\left(S_{g, n}\right)$ contains an infinite, disjoint collection of $\epsilon$-balls. This contradicts the finite volume hypothesis.

For Theorem 1.3, we illustrate that the existence of a $\operatorname{Mod}(S)$-equivariant complete Riemannian metric on Teich $(S)$ of pinched negative curvature puts even stronger restrictions on an isometric action of $\operatorname{Mod}(S)$.

Proof: (of Theorem 1.3). If $\mathcal{M}_{g, n}$ did admit such a metric, then lifting this metric to the universal cover Teich $\left(S_{g, n}\right)$ gives a properly discontinuous, isometric action of $\operatorname{Mod}\left(S_{g, n}\right)$ on a complete, 1-connected, pinched negatively curved manifold $X=\operatorname{Teich}\left(S_{g, n}\right)$.

Since $3 g-3+n \geq 2$, there exists a subgroup $N$ of $\operatorname{Mod}\left(S_{g, n}\right)$ generated by Dehn twists as above, with elements conjugate in $\operatorname{Mod}\left(S_{g, n}\right)$, and with the property that $N$ is not virtually nilpotent (since it contains, for example, noncyclic free subgroup (see, e.g. [FLM])). Note that in case $g=0$, the elements $g_{i}$ can be taken to be Dehn twists about curves which surround two punctures (hence are separating), in particular they are conjugate in $\operatorname{Mod}\left(S_{g, n}\right)$.

As $X$ is $\delta$-hyperbolic, the exact same argument as in the proof of Theorem 1.2 gives some $x \in X$ which is fixed by each generator $g_{i}$.

Fix any horosphere $H$ based at $x$, and fix a basepoint $s \in H$. Then $d\left(s, g_{i} s\right) \leq C_{H}$ for some constant $C_{H}$ for each generator $g_{i}$. As the sectional curvature of $X$ is pinched between two negative constants, we may find a horosphere $H$ based at $x \in \partial X$ so that $C_{H}$ is as small as we want; the key point is that the pinching of the curvatures gives a definite (exponential) rate at which geodesic rays asymptotic to $x$ converge. Choosing $H$ so that $C_{H}$ is smaller than the Margulis constant for $X$ (which depends only on $\operatorname{dim}(X)$ and on the pinching constants of the sectional curvatures) and applying the Margulis Lemma (see, e.g. [BGS]), it follows that $N$ contains a nilpotent subgroup of finite index, a contradiction.

Remark. Theorem 1.3 may also be deduced (though not in some of the low genus cases) from the topological structure of the end of $\mathcal{M}_{g, n}$. On the one 
hand, every end of a finite volume manifold of pinched negative curvature is homeomorphic to the product of a compact nilmanifold and $[0, \infty)$; this is essentially the Margulis Lemma (see [BGS]). On the other hand, it seems to be well-known (see, e.g. [Fa]) that the entire orbifold fundamental group of $\mathcal{M}_{g, n}$ is carried by its end; in particular the fundamental group of the end is not virtually nilpotent. This argument actually shows that no finite cover of $\mathcal{M}_{g, n}$ admits a complete, finite volume Riemannian metric of pinched negative curvature.

\section{Quasi-flats in the pants complex}

Let $S$ be a surface of genus $g$ with $n$ boundary components. A subsurface $R \subset S$ is a compact connected embedded surface lying in $S$. The subsurface $R$ is essential if its boundary components are homotopically essential in $S$.

We say $S$ decomposes into essential subsurfaces $R_{1}, \ldots, R_{k}$ if each $R_{j}$ may be modified by an isotopy so that they are pairwise disjoint and $S-$ $R_{1} \sqcup \ldots \sqcup R_{k}$ is a collection of open annular neighborhoods of simple closed curves on $S$, each isotopic to a boundary component of $R_{j}$.

Let $r(S)$ denote the maximum number $k$ in any decomposition of $S$ into essential subsurfaces $R_{1}, \ldots, R_{k}$ such that each $R_{j}, j=1, \ldots, k$ has genus at least one, or at least four boundary components. Then $r(S)$ is greatest integer less which is at most $(d(S)+1) / 2$.

Theorem 4.1 The complex $C_{\mathbf{P}}(S)$ contains a quasi-flat of dimension $r(S)$.

Proof: The surface $S$ decomposes into subsurfaces

$$
R_{1}, \ldots, R_{r(S)}, T
$$

so that $d\left(R_{j}\right)=1$ for each $j$ and either $T$ is empty or $d(T)=0$. Let $n=r(S)$. We describe a quasi-isometric embedding of the Cayley graph for $\mathbb{Z}^{n}$ with the standard generators into the complex $C_{\mathbf{P}}(S)$.

Let $c_{j}$ be a vertex in the curve complex $\mathcal{C}\left(R_{j}\right)$. Then together with the core curves of the open annuli in $S-R_{1} \sqcup \ldots \sqcup R_{r(S)} \sqcup T$, the curves $c_{j}$ form a pants decomposition $P=P\left(c_{1}, \ldots, c_{n}\right)$ of $S$.

We let $g_{j}: \mathbb{Z} \rightarrow \mathcal{C}\left(R_{j}\right)$ be a geodesic so that $g_{j}(0)=c_{j}$. Note that $\mathcal{C}\left(R_{j}\right)$ is the Farey graph, so we may take any bi-infinite geodesic $g_{j}$ in $\mathcal{C}\left(R_{j}\right)$.

We claim that the embedding

$$
Q: \mathbb{Z}^{n} \rightarrow C_{\mathbf{P}}(S)
$$


defined by

$$
Q\left(k_{1}, \ldots, k_{n}\right)=P\left(g_{1}\left(k_{1}\right), \ldots, g_{n}\left(k_{n}\right)\right)
$$

is a quasi-isometry, whose constants depend only on $S$.

Let $\vec{k}=\left(k_{1}, \ldots, k_{n}\right)$ and $\vec{l}=\left(l_{1}, \ldots, l_{n}\right)$. Since elementary moves along $g_{j}$ can be made independently in each $R_{j}$, we have

$$
d_{\mathbf{P}}(Q(\vec{k}), Q(\vec{l})) \leq \sum_{j=1}^{n}\left|l_{j}-k_{j}\right|=d_{\mathbb{Z}^{n}}(\vec{k}, \vec{l})
$$

which shows that $Q$ is 1-Lipschitz.

Given $R_{j}$, the projection $\pi_{R_{j}}(Q(\vec{k}))$ to $R_{j}$ described in $\S 2.3$ simply picks out the curve $g_{j}\left(k_{j}\right)$ so we have

$$
\pi_{R_{j}}(Q(\vec{k}))=g_{j}\left(k_{j}\right)
$$

Thus, the projection distance

$$
d_{R_{j}}(Q(\vec{k}), Q(\vec{l}))=d_{R_{j}}\left(g_{j}\left(k_{j}\right), g_{j}\left(l_{j}\right)\right)
$$

which is simply $\left|k_{j}-l_{j}\right|$ since $g_{j}$ is a geodesic in $\mathcal{C}\left(R_{j}\right)$.

By Theorem 6.12 of [MM2], there exists $M_{0}=M_{0}(S)$ so that for all $M \geq M_{0}$ there exist constants $K_{0}$ and $K_{1}$ so that if we let $P_{\vec{k}}=Q(\vec{k})$ and $P_{\vec{l}}=Q(\vec{l})$ then we have the inequality

$$
\sum_{\substack{Y \subseteq S \\ d_{Y}\left(\pi_{Y}\left(P_{\vec{k}}, \pi_{Y}\left(P_{\vec{l}}\right)\right)>M\right.}} d_{Y}\left(P_{\vec{k}}, P_{\vec{l}}\right) \leq K_{0} d_{\mathbf{P}}\left(P_{\vec{k}}, P_{\vec{l}}\right)+K_{1} .
$$

But the left-hand-side of the inequality is bounded below by

$$
\max _{j}\left|k_{j}-l_{j}\right| \geq \frac{\sum_{j}\left|k_{j}-l_{j}\right|}{n} .
$$

Thus, $Q$ is a quasi-isometric embedding.

\section{The case of low genus}

In the case where $d(S)=1$, where $\operatorname{int}(S)$ is homeomorphic to a punctured torus or four-times-punctured sphere, the pants complex $C_{\mathbf{P}}(S)$ is identified with the curve complex $\mathcal{C}(S)$, which is Gromov-hyperbolic (see [MM1]).

Together with the previous section, this observation leaves one case unattended, namely that when $d(S)=2$. In this case, $\operatorname{int}(S)$ is homeomorphic either to a doubly-punctured torus, or a five-times-punctured sphere. In this section we prove the following. 
Theorem 5.1 Let $S$ be such that $d(S)$ equals 1 or 2 . Then the WeilPetersson metric on Teich $(S)$ is Gromov-hyperbolic.

The case $d(S)=1$ is proven in [MM1], since $\mathcal{C}(S)=C_{\mathbf{P}}(S)$ in this case. Thus we are left to treat the case when $d(S)=2$. In this case, we apply results of the second author which, although initially phrased in the context of groups with their word metrics, apply to general metric spaces.

Our argument will employ the notion of relative hyperbolicity developed in $[\mathrm{Fa}]$. In essence, a metric space is relatively hyperbolic relative to a collection of subsets if the result of crushing those subsets to have diameter 1 is a hyperbolic metric space. In the case $d(S)=2$, we will show that $C_{\mathbf{P}}(S)$ is relatively hyperbolic relative to regions consisting of pants decompositions containing a single curve. Since, in this case, such regions are themselves hyperbolic, it is possible to establish Gromov-hyperbolicity $C_{\mathbf{P}}(S)$ by showing that paths in $C_{\mathbf{P}}(S)$ that determine quasi-geodesics in the relative space satisfy certain boundedness properties with respect to their trajectories through these regions (this is the bounded region penetration property, below).

Remark: One can prove theorem 5.1 by directly demonstrating a thintriangles condition after replacing geodesics in $C_{\mathbf{P}}(S)$ by the hierarchies of [MM2] (this was our original approach to the argument). We have chosen instead to employ the theory relative hyperbolicity as it is more familiar, and unifies these cases with the higher genus cases. Indeed, when $d(S)>2$ the natural regions with respect to which $C_{\mathbf{P}}(S)$ is relatively hyperbolic (sub-graphs of pairs of pants containing a given curve $\alpha$ ) are not themselves hyperbolic; they are the quasi-flats of the previous section.

Relative hyperbolicity. Let $(X, d)$ be a geodesic metric space, and let $H_{\alpha}$ be a collection of connected subsets of $X$, with index $\alpha$ in an index set $A$. Then the electric space $\widehat{X}$ relative to the regions $\left\{H_{\alpha}\right\}$ is obtained by collapsing each region to have diameter one, as follows (see [Fa, Sec. 3]). Adjoin to the space $X$ a single point $c_{\alpha}$ for each $\alpha \in A$ by connecting $c_{\alpha}$ to each point of $H_{\alpha}$ by a segment of length $1 / 2$. Let $\widehat{X}$ denote the resulting path-metric space and let $d_{e}(.,$.$) denote path distance in \widehat{X}$. Given a path $w$ in $X$ we obtain a path in $\widehat{X}$ by replacing segments where $w$ travels in $H_{\alpha}$ with a path joining the endpoints of the segment to $c_{\alpha}$. As in [Fa], we denote this path-replacement procedure by $X \rightarrow \widehat{X}$ or $w \mapsto \hat{w}$. We denote by $I(w)$ the initial point of $w$ and by $T(w)$ the terminal point of $w$. The points $I(w)$ and $T(w)$ depend on the choice of parameterization.

If $\hat{w}$ is a (k-quasi) geodesic in $\widehat{X}$ we say $w$ is a relative ( $k$-quasi) geodesic in $X$. If a path $w$ in $X$ (or $\hat{w}$ in $\widehat{X}$ ) passes through some region $H_{\alpha}$ we 
say it penetrates $H_{\alpha}$. A path $w \in X$ (or $\hat{w}$ in $\widehat{X}$ ) has no backtracking if for every region $H_{\alpha}$ that $\hat{w}$ penetrates, once it leaves $H_{\alpha}$ it never returns. The space $X$ is hyperbolic relative to $\left\{H_{\alpha}\right\}_{\alpha \in A}$ if the electric space $\widehat{X}$ is Gromov hyperbolic.

In the pants complex, consider following collection of regions: for each $\alpha \in \mathcal{C}(S)$ let

$$
H_{\alpha}=\left\{P \in C_{\mathbf{P}}(S) \mid \alpha \in P\right\} .
$$

Then we have the following theorem (cf. [MM1, Thms. 1.2, 1.3]).

Lemma 5.2 The graph $C_{\mathbf{P}}(S)$ is hyperbolic relative to the regions $\left\{H_{\alpha}\right\}$.

Proof: It suffices to show that the electric space $\widehat{C_{\mathbf{P}}(S)}$ with respect to the regions $\left\{H_{\alpha}\right\}$ is quasi-isometric to the curve complex $\mathcal{C}(S)$, which is Gromov-hyperbolic by [MM1, Thm. 1.1].

To see this, let $\Gamma=C_{\mathbf{P}}(S)$, let $\widehat{\Gamma}$ be the electric space associated to the regions $\left\{H_{\alpha}\right\}$, and let $c_{\alpha}$ be the point added to $\Gamma$ at distance $1 / 2$ from each point of $H_{\alpha}$ to form $\widehat{\Gamma}$. Consider the mapping

$$
q: \mathcal{C}^{0}(S) \rightarrow \widehat{\Gamma}
$$

from the zero-skeleton of $\mathcal{C}(S)$ to $\Gamma$ obtained by setting $q(\alpha)=c_{\alpha}$. Note that given a pants decomposition $P$, if $\beta$ is an element of $P$ then $P$ lies a distance $1 / 2$ from $c_{\beta}$, so the image $q\left(\mathcal{C}^{0}(S)\right)$ is $1 / 2$-dense.

Moreover, we have $d_{\mathcal{C}(S)}(\alpha, \beta)=1$ if and only if there is a $P$ for which $\alpha \in P$ and $\beta \in P$. But $\alpha \cup \beta \subset P$ holds if and only if the regions $H_{\alpha}$ and $H_{\beta}$ intersect, which holds if and only if

$$
d_{\widehat{\Gamma}}\left(c_{\alpha}, c_{\beta}\right)=1 .
$$

Thus, the map $q$ is 1 -bi-Lipschitz, and since the image is $1 / 2$-dense we may construct a 2-Lipschitz inverse to $q$. Thus, $q$ a quasi-isometry.

Bounded region penetration. We now recall results of $[\mathrm{Fa}]$ detailing a criterion on relative quasi-geodesics that will serve to ensure hyperbolicity of $C_{\mathbf{P}}(S)$ when $d(S)=2$. The following definition is analogous to the "bounded coset penetration property" in $\S 3.3$ of $[\mathrm{Fa}]$.

Definition 5.3 (bounded region penetration) The pair $\left(X,\left\{H_{\alpha}\right\}\right)$ satisfies the bounded region penetration property if, for every $P \geq 1$ there is a constant $c=c(P)>0$ so that if $u$ and $w$ are relative $P$-quasi-geodesics without backtracking so that the initial and terminal points of $u$ and $w$ satisfy $d_{X}(I(u), I(w)) \leq 1$ and $d_{X}(T(u), T(w)) \leq 1$ then the following holds: 
1. If $u$ penetrates a region $H_{\alpha}$ but $w$ does not penetrate $H_{\alpha}$, then $u$ travels an $X$-distance at most $c$ in $H_{\alpha}$.

2. If both $u$ and $w$ penetrate a region $H_{\alpha}$ then the points at which $u$ and $w$ first enter $H_{\alpha}$ lie an $X$-distance at most $c$ from one another and likewise for the exit points.

An important application is the following theorem in which bounded region penetration is used to bootstrap from hyperbolicity relative to hyperbolic regions to hyperbolicity of the original metric space.

Theorem 5.4 Suppose $X$ is hyperbolic relative to the regions $\left\{H_{\alpha}\right\}$ and that the pair $\left(X,\left\{H_{\alpha}\right\}\right)$ has the bounded region penetration property. Then if the regions $H_{\alpha}$ are themselves $\delta$-hyperbolic metric spaces for some $\delta>0$, then $X$ is a Gromov-hyperbolic metric space.

Proof: (of Theorem 5.4). Theorem 5.4 is simply a recasting of the remark following [Fa, Thm. 3.8] from groups to general metric spaces. The proof works verbatim in this case, with the following addition: one replaces the use of the theorem that linear isoperimetric inequality for a group implies that the group is Gromov-hyperbolic by the corresponding theorem for metric spaces with a well-defined notion of area. Such a theorem is proven by Bowditch in [Bowd]; in this case one can use for area the combinatorial area of the simplicial complex whose 1-skeleton is the pants graph $C_{\mathbf{P}}(S)$ and whose 2-cells consist of five types of loops with $3,4,5$, and 6 edges, and no other edges between vertices (this is a variant of the 2-complex studied by Hatcher-Thurston in [HT]. It is shown to be simply connected in [HLS, Thm. D]). One may verify that Bowditch's proof extends to the locally infinite case.

Proof: (of Theorem 5.1). The condition $d(S)=2$ implies that each pants decomposition of $S$ is built from exactly two disjoint simple closed curves on $S$.

Lemma 5.5 Suppose $d(S)=2$. Then there is a $\delta$ so that for each $\alpha \in \mathcal{C}(S)$, the region $H_{\alpha}$ is $\delta$-hyperbolic.

Proof: Given $\alpha \in \mathcal{C}(S)$, let $Y_{\alpha}$ denote the connected component of the complement of an embedded open annular neighborhood of $\alpha$ for which $d\left(Y_{\alpha}\right)=1$. Then the region $H_{\alpha}$ is isometric to the curve complex $\mathcal{C}\left(Y_{\alpha}\right)$. Again, [MM1, Thm. 1.1] implies that $H_{\alpha}$ is hyperbolic. 
To prove Theorem 5.1, then, it suffices to prove that when $d(S)=2$, the pair $\left(C_{\mathbf{P}}(S),\left\{H_{\alpha}\right\}\right)$ has the bounded region penetration property. To this end, let $u$ and $w$ be two relative $P$-quasi-geodesics in $C_{\mathbf{P}}(S)$ without backtracking, so that $d_{\mathbf{P}}(I(u), I(w)) \leq 1$ and $d_{\mathbf{P}}(T(u), T(w)) \leq 1$. Let $H_{\alpha}$ be a region which $u$ penetrates but $w$ does not. Being relative $P$-quasigeodesics in the relatively hyperbolic space $\left(C_{\mathbf{P}}(S),\left\{H_{\alpha}\right\}\right)$, it follows form the definition that the projections $\hat{u}$ and $\hat{w} D$-fellow-travel in the electric space $\widehat{C_{\mathbf{P}}(S)}$ for some $D>0$ depending only on $P$. For simplicity of notation let $\Gamma=C_{\mathbf{P}}(S)$ and let $\widehat{\Gamma}$ be the associated electric space relative to the regions $\left\{H_{\alpha}\right\}$.

We observe that in our circumstances, the path replacement $u \mapsto \hat{u}$ can be viewed as producing an explicit path in $\mathcal{C}(S)$ from a path $u$ in $\Gamma$. Since $d(S)=2$, each pants decomposition in of $S$ has two elements, so a path $u$ in $\Gamma$ is a sequence of edges in $\mathcal{C}(S)$ each of which is joined to the previous one at one of its two endpoints. Let $\tilde{u}$ denote the path in $\mathcal{C}(S)$ obtained by removing all but the first and last edges in any sequence of consecutive edges in $u$ that all contain a single vertex. The path $\tilde{u}$ in $\mathcal{C}(S)$ has image $\hat{u}$ under the quasi-isometry $q$ (up to segments of length $1 / 2$ at the endpoints).

To employ the extra information the curve complex provides, we work with $\tilde{u}$ and $\tilde{w}$ rather than $\hat{u}$ and $\hat{w}$. The condition that $u$ is a relative $P$-quasigeodesic without backtracking simply means that the path $\tilde{u}$ is a $P$-quasigeodesic in $\mathcal{C}(S)$ that never repeats a vertex. Proving that $\left(\Gamma,\left\{H_{\alpha}\right\}\right)$ satisfies bounded region penetration property, then, reduces to verifying that for paths $u$ and $w$ in $\Gamma$ whose for which $d_{\Gamma}(I(u), I(w)) \leq 1$ and $d_{\Gamma}(T(u), T(w)) \leq$ 1 , and whose corresponding paths $\tilde{u}$ and $\tilde{w}$ are $P$-quasi-geodesics without backtracking we have:

$1^{\prime}$ If $\tilde{u}$ encounters a vertex $v_{\alpha}$ that $\tilde{w}$ avoids, the vertices $v_{\beta}$ and $v_{\gamma}$ adjacent to $v_{\alpha}$ on $\tilde{u}$ have distance

$$
d_{Y_{\alpha}}\left(v_{\beta}, v_{\gamma}\right)<c
$$

in the subsurface $Y_{\alpha}$.

$2^{\prime}$ If $\tilde{u}$ and $\tilde{w}$ each encounter a vertex $v_{\alpha}$, then the vertices $v_{\beta} \in \tilde{u}$ and $v_{\beta^{\prime}} \in \tilde{w}$ just prior to the encounter with $v_{\alpha}$ satisfy

$$
d_{Y_{\alpha}}\left(v_{\beta}, v_{\beta^{\prime}}\right)<c
$$

and likewise for the points $v_{\gamma}$ and $v_{\gamma^{\prime}}$ on $\tilde{u}$ and $\tilde{w}$ just following the encounter with $v_{\alpha}$. 
(For the remainder of this section we will denote by $v_{\alpha}$ the vertex in $\mathcal{C}(S)$ corresponding to the simple closed curve $\alpha$ on $S$ to avoid notational confusion). To see property $\left(1^{\prime}\right)$ implies property (1) above, note that the condition that $\left\{v_{\beta}, v_{\alpha}, v_{\gamma}\right\}$ is a sub-segment of $\tilde{u}$ implies that $P=\left\{v_{\alpha}, v_{\beta}\right\}$ and $P^{\prime}=\left\{v_{\alpha}, v_{\gamma}\right\}$ are the pants decompositions along the path $u$ where $u$ enters and exits the region $H_{\alpha}$. If $d_{Y_{\alpha}}\left(v_{\beta}, v_{\gamma}\right)<c$ then there is a sequence of pants decompositions joining $P$ to $P^{\prime}$ of length at most $c$ given by taking a geodesic

$$
\left\{v_{\beta}=v_{0}, \ldots, v_{N}=v_{\gamma}\right\}
$$

joining $v_{\beta}$ to $v_{\gamma}$ in $\mathcal{C}\left(Y_{\alpha}\right)$ and taking the sequence of pants decompositions to be $\left\{P_{j}=\left\{v_{\alpha}, v_{j}\right\}\right\}_{j}$. One argues similarly that property $\left(2^{\prime}\right)$ implies property $(2)$.

We now verify that properties $\left(1^{\prime}\right)$ and $\left(2^{\prime}\right)$ hold. Since $\tilde{u}$ does not backtrack, we may choose points $x$ and $y$ on $\tilde{u}$ on either side of the vertex $v_{\alpha}$ as follows. Either $x$ is an endpoint of $\tilde{u}$ or $x$ lies at distance $2 D$ in $\mathcal{C}(S)$ from the vertex $v_{\beta}$ adjacent on $\tilde{u}$ to $v_{\alpha}$ whichever is closer along $\tilde{u}$. The point $y$ is either an endpoint of $\tilde{u}$ or $y$ lies at distance $2 D$ along $\tilde{u}$ from the vertex $v_{\gamma}$ adjacent to $v_{\alpha}$ whichever is closer along $\tilde{u}$. There is then a path $p$ on $\tilde{w}$ (which does not encounter $v_{\alpha}$ ) from the nearest point to $x$ on $\tilde{w}$ to the nearest point to $y$. Letting $p_{x}$ be the shortest path joining $x$ to $\tilde{w}$ and letting $p_{y}$ be the shortest path joining $y$ to $\tilde{w}$ in $\mathcal{C}(S)$, the concatenation

$$
q=p_{x} \circ p \circ p_{y}
$$

is a path in $\mathcal{C}(S)$ that avoids the vertex $v_{\alpha}$. Moreover, the path $q$ has length at most $8 D$.

Letting $q_{x}$ be the path along $\tilde{u}$ joining $x$ to $v_{\beta}$, and letting $q_{y}$ be the path along $\tilde{u}$ joining $y$ to $v_{\gamma}$, we have a path

$$
r=q_{x} \circ p \circ q_{y}
$$

of length at most $12 D$ that avoids the vertex $v_{\alpha}$ entirely.

The path $r$ describes a path in the curve complex $\mathcal{C}(S)$ so that each vertex along the interior of $r$ corresponds to a curve that either lies in $\mathcal{C}\left(Y_{\alpha}\right)$ or intersects $\partial Y_{\alpha}$. It follows from lemma 2.2 that any two consecutive vertices $z$ and $z^{\prime}$ on $r$ satisfy

$$
d_{Y_{\alpha}}\left(z, z^{\prime}\right) \leq 2
$$

Thus we have the bound

$$
d_{Y_{\alpha}}\left(v_{\alpha}, v_{\beta}\right) \leq 24 D
$$


A similar argument proves property (2) of the bounded region penetration property holds. Choose a point $x$ on $\tilde{u}$ so that either $x$ is the first vertex of $\tilde{u}$ or $x$ is at distance $2 D$ along $\tilde{u}$ from $v_{\beta}$, whichever is closer along $\tilde{u}$ to $v_{\beta}$. By fellow traveling, there is a point $y$ on $\tilde{w}$ and a path $p$ of length at most $D$ joining $x$ to $y$ in $\mathcal{C}(S)$. The path along $\tilde{w}$ joining $y$ to the last vertex $v_{\eta}$ prior to $v_{\alpha}$ along $\tilde{w}$ has length at most $2 D(1+P)$ in $\mathcal{C}(S)$, so there is a path $r$ in $\mathcal{C}(S)$ of total length bounded by $3 D+2 D(1+P)$ joining $v_{\beta}$ to $v_{\eta}$ that does not hit $v_{\alpha}$ in its interior. Again, by lemma 2.2 any two consecutive vertices $z$ and $z^{\prime}$ on $r$ have the property that

$$
d_{Y_{\alpha}}\left(z, z^{\prime}\right) \leq 2
$$

so we have

$$
d_{Y_{\alpha}}\left(v_{\alpha}, v_{\eta}\right) \leq 2(3 D+2 D(1+P)) .
$$

The same argument proves that points on $v_{\alpha}^{\prime}$ and $v_{\eta}^{\prime}$ adjacent to $v_{\alpha}$ where $\tilde{u}$ and $\tilde{w}$ depart from $v_{\alpha}$ also have bounded distance $d_{Y_{\alpha}}\left(v_{\alpha}^{\prime}, v_{\eta}^{\prime}\right)$.

Having verified that properties $\left(1^{\prime}\right)$ and $\left(2^{\prime}\right)$ hold, we conclude that the pair $\left(\Gamma,\left\{H_{\alpha}\right\}\right)$ has the bounded region penetration property. The theorem follows from theorem 5.4.

\section{Questions}

We close the paper with some natural questions.

Question 6.1 (McMullen) Does $\mathcal{M}_{g, n}$ admit a complete, nonpositively curved Riemannian metric?

McMullen's Kähler metric on $\mathcal{M}_{g, n}$ is complete but not nonpositively curved, while the Weil-Petersson metric is nonpositively curved but is not complete. Is there a possible compromise?

Question 6.2 What is the geometric rank of

- the Weil-Petersson metric?

- the Teichmüller metric?

- the mapping class group? 
Theorem 1.4 gives the lower bound $d(S) / 2$ to the rank of the Weil-Petersson metric, while [Min] and [FLM] establish the lower bound $d(S)$ for the rank of the Teichmüller metric and the mapping class group respectively.

The answers to these rank questions seem to be essential to understanding quasi-isometric rigidity questions in Teichmüller space and the mapping class group.

Question 6.3 If $\operatorname{int}(S)$ is homeomorphic to a doubly-punctured torus or 5times-punctured sphere, are the sectional curvatures of the Weil-Petersson metric bounded away from zero?

Were the (geodesically convex) Weil-Petersson metric to have curvature pinched from above by a negative constant, its Gromov-hyperbolicity in this case would be an immediate consequence.

\section{References}

[BGS] W. Ballman, M. Gromov, and V. Schroeder. Manifolds of Nonpositive Curvature. Birkhauser, 1985.

[Be] M. Bestvina. Questions in Geometric Group Theory. Available online at http://www.math.utah.edu/ bestvina.

[Bowd] B. H. Bowditch. Notes on Gromov's hyperbolicity criterion. In E. Ghys, A Haefliger, and A. Verjovsky, editors, Group Theory from a Geometrical Viewpoint, pages 64-167. World Scientific, 1991.

[Br] J. Brock. The Weil-Petersson metric and volumes of 3-dimensional hyperbolic convex cores. Preprint, 2001.

[EF] A. Eskin and B. Farb. Quasi-flats and rigidity in higher rank symmetric spaces. Journal Amer. Math. Soc. 10(1997), 653-692.

[Fa] B. Farb. Relatively hyperbolic groups. Geom. \& Funct. Anal. 8(1998), 1-31.

[Fa] B. Farb. A primer on mapping class groups. In preparation.

$[\mathrm{FF}] \quad$ B. Farb and J. Franks. Groups of homeomorphisms of onemanifolds, I: actions of nonlinear gropus. Preprint (2001). 
[FLM] B. Farb, A. Lubotzky, and Y. Minsky. Rank one phenomena for mapping class groups. Duke Math. J. 106(2001), 581-597.

[GH] E. Ghys and P. de la Harpe, editors. Sur les groupes hyperboliques d'ápres Mikhael Gromov, volume 83 of Progress in Mathematics. Birkhauser, 1990.

[Grom] M. Gromov. Asymptotic invariants of infinite groups. In G. Niblo and M. Roller, editors, Geometric Group Theory, volume 182 of LMS Lecture Notes. Cambridge Univ. Press, 1993.

[HLS] A. Hatcher, P. Lochak, and L. Schneps. On the Teichmüller tower of mapping class groups. J. reine angew. Math. 521(2000), 1-24.

[HT] A. Hatcher and W. Thurston. A presentation for the mapping class group. Topology 19(1980), 221-237.

[Iv1] N. V. Ivanov. Subgroups of Teichmüller Modular Groups, volume 115 of AMS Translations of Math. Monographs. American Mathematical Society, 1992.

[Iv2] N. V. Ivanov. Mapping class groups. Preprint.

[KL] B. Kleiner and B. Leeb. Rigidity of quasi-isometries for symmetric spaces and Euclidean buildings. Publ. IHES 86(1997), 115-197.

[Kr] S. Kravetz. On the geometry of Teichmller spaces and the structure of their modular groups. Ann. Acad. Sci. Fenn. 278(1959), 35 pp.

[Mas1] H. Masur. On a class of geodesics in Teichmüller space. Annals of Math. 102(1975), 205-221.

[Mas2] H. Masur. The extension of the Weil-Petersson metric to the boundary of Teichmüller space. Duke Math. J. 43(1976), 623-635.

[MM1] H. Masur and Y. Minsky. Geometry of the complex of curves I: hyperbolicity. Invent. Math. 138(1999), 103-149.

[MM2] H. Masur and Y. Minsky. Geometry of the complex of curves II: hierarchical structure. Geom. \& Funct. Anal. 10(2000), 902-974.

[MW] H. Masur and M. Wolf. Teichmüller space is not Gromov hyperbolic. Ann. Acad. Sci. Fenn. 20(1995), 259-267. 
[MP1] J. McCarthy and A. Papadopoulos. The mapping class group and a theorem of Masur-Wolf. Topology Appl. 96(1996), 75-84.

[MP2] J. McCarthy and A. Papadopoulos. The visual sphere of Teichmller space and a theorem of Masur-Wolf. Ann. Acad. Sci. Fenn. Math. 24(1999), 147-154.

[Mc] C. McMullen. The moduli space of Riemann surfaces is Kähler hyperbolic. Annals of Math. 151(2000), 327-357.

[Min] Y. Minsky. Quasi-projections in Teichmüller space. J. Reine Angew. Math. 473(1996), 121-136.

[Wol] S. Wolpert. Noncompleteness of the Weil-Petersson metric for Teichmüller space. Pacific J. Math. 61(1975), 573-577.

Math Department, University of Chicago, 5734 S. University Ave., Chicago, IL 60637

EMAIL: BROCK@MATH.UCHICAGO.EDU, FARB@MATH.UCHICAGO.EDU 